BULL. AUSTRAL. MATH. SOC.

VOL. $23(1981), 307-311$.

\title{
ON RINGS WITH NIL COMMUTATOR IDEAL
}

\author{
Hazar ABu-Khuzam
}

\begin{abstract}
Let $R$ be a ring in which for each $x, y$ in $R$ there exists a positive integer $n=n(x, y)$ such that $(x y)^{n}-(y x)^{n}$ is in the center of $R$. Then $R$ has a nil commutator ideal.
\end{abstract}

A theorem of Belluce, Herstein and Jain [2] states that, if $R$ is a ring in wich for each $x, y$ in $R$ there exists integers $m=m(x, y) \geq 1, n=n(x, y) \geq 1$ such that $(x y)^{m}=(y x)^{n}$, then the commutator ideal of $R$ is nil. Our objective is to generalize the above result for the case where $m(x, y)=n(x, y)$. Indeed, we prove that, if $R$ is a ring in which for each $x, y$ in $R$ there exists an integer $n=n(x, y) \geq 1$ such that $(x y)^{n}-(y x)^{n}$ is in the center of $R$, then $R$ has a nil commutator.

In preparation for the proofs of our main theorem, we first consider the following lemas. Throughout, $R$ will denote a ring, $Z$ will denote the center of $R$, and $J$ the jacobson radical of $R$. We use the standard notation $[x, y]=x y-y x$.

The first two lemas are known and we omit their proofs.

LEMMA 1. If $[x, y]$ commutes with $x$, then $\left[x^{k}, y\right]=k x^{k-1}[x, y]$.

LEMMA 2. Let $d$ be a derivation of $R$. If $x \in R$ is such that $d^{2}(x)=0$ then $d^{k}\left(x^{k}\right)=k !(d(x))^{k}$ for all $k \geq 1$.

Received 18 November 1980. 
LEMMA 3. If $R$ is a ring in which for each $x, y$ in $R$, there exists an integer $n=n(x, y) \geq 1$ such that $(x y)^{n}-(y x)^{n} \in Z$. Then for each $a \in J, x \in R$ there exist integers $n=n(x, a) \geq 1$ and $m=m(x, a) \geq 1$ such that $(4 m) !\left[a, x^{2 n}\right]^{4 m}=0$.

Proof. Let $a \in J, x \in R,(1+a)$ is formally invertible ( $R$ need not have an identity element). Using the hypothesis for the elements $(1+a) x$ and $x(1+a)^{-1}$, there exists an integer $n \geq 1$ such that

$$
\left((1+a) x^{2}(1+a)^{-1}\right)^{n}-x^{2 n} \in z \text {. }
$$

Thus

$$
\begin{aligned}
(1+a) x^{2 n}-x^{2 n}(1+a) & =(1+a)\left((1+a) x^{2 n}(1+a)^{-1}-x^{2 n}\right), \\
\left((1+a) x^{2 n}-x^{2 n}(1+a)\right)(1+a) & =(1+a)\left((1+a) x^{2 n}-x^{2 n}(1+a)\right) .
\end{aligned}
$$

Hence

$$
\left(a x^{2 n}-x^{2 n} a\right) a=a\left(a x^{2 n}-x^{2 n} a\right), a \in J, x \in R
$$

Let $d(y)=a y-y a \cdot d$ is a derivation of $R$. Using (1), $d^{2}\left(x^{2 n}\right)=0$. Applying (1) for $x^{4 n}$ instead of $x$, there exists an integer $m \geq 1$ such that

$$
\left(a\left(x^{4 n}\right)^{2 m}-\left(x^{4 n}\right)^{2 m} a\right) a=a\left(a\left(x^{4 n}\right)^{2 m}-\left(x^{4 n}\right)^{2 m} a\right)
$$

Thus, $d^{2}\left(x^{8 m n}\right)=0$. Hence, by Lemma 2,

$$
0=d^{4 m}\left(\left(x^{2 n}\right)^{4 m}\right)=(4 m) !\left(d\left(x^{2 n}\right)\right)^{4 m}
$$

and so $(4 m) !\left[a, x^{2 n}\right]^{4 m}=0$.

Theorem 1 below is proved in [1] and we omit its proof here.

THEOREM 1. If $R$ is a semisimple ring in which, for each $x, y$ in $R$ there exists an integer $n=n(x, y) \geq 1$ such that $(x y)^{n}=(y x)^{n} \in Z$. Then $R$ is commutative.

THEOREM 2. Let $R$ be a ring in which, for each $x, y$ in $R$ there exists an integer $n=n(x, y) \geq 1$ such that $(x y)^{n}-(y x)^{n} \in Z$. Then the commutator ideal of $R$ is nil. Equivalently, if $R$ has no nonzero 
nil ideals then $R$ is commutative.

Proof. To prove that the commutator ideal of $R$ is nil it is enough to show that if $R$ has no nonzero nil ideals then it is commutative. So we suppose that $R$ has no nonzero nil ideals. Then $R$ is a subdirect product of prime rings $R_{\alpha}$, having no nonzero nil ideals, such that in each $R_{\alpha}$ there is a nonnilpotent element $b_{\alpha}$ in which $b_{\alpha}^{t(I)} \in I$ for every nonzero ideal $I$ of $R_{\alpha}$. Clearly, $R_{\alpha}$ satisfies the condition $(x y)^{n}-(y x)^{n} \in z_{\alpha}\left[\right.$ center of $\left.R_{\alpha}\right]$. So we may assume that $R$ is a prime ring, having no nonzero nil ideals, in which there is a nonnilpotent element $b \in R$ such that $b^{t(I)} \in I$ for all nonzero ideals $I$ of $R$. We may assume that $J \neq 0$, otherwise the result follows from Theorem 1 . If $\operatorname{char} R=p \neq 0$, then, by (1), for any $x \in R$ and $a \in J$, there exists an integer $n=n(a, x) \geq 1$ such that

$$
\left[a,\left[a, x^{2 n}\right]\right]=0 \text {. }
$$

Hence, by Lemma 1 ,

$$
\left[a^{p}, x^{2 n}\right]=p a^{p-1}\left[a, x^{2 n}\right]=0
$$

So for any $x, y \in J,\left[x^{p}, y^{2 n}\right]=0$. This implies by [3] that $J$ is commutative, and therefore $R$ is commutative since it is prime and has a nonzero comutative ideal [4].

So we may assume that $\operatorname{char} R=0$, and since $R$ is prime with char $R=0, R$ is torsion-free.

CLAIM 1. Every zero divisor in $R$ is nilpotent.

To prove Claim I, suppose that $a c=0$, with $a \neq 0$ and $c$ nonnilpotent. Let $A=\left\{x \in R: x e^{r}=0\right.$ for some $\left.r \geq 1\right\}$ and $B=\left\{x \in R: c^{8} x=0\right.$ for some $\left.\theta \geq 1\right\}$. Then $A$ is a left ideal of $R$, and $B$ is a right ideal. $A \neq 0$ since $0 \neq a \in A$. If $x \in A$, then $x c^{r}=0$ for some $r \geq 1$, and hence $\left(c^{r} x\right)^{2}=0$. By hypothesis, there exists an integer $n \geq 1$ such that $\left(c^{r}\left(x+c^{n}\right)\right)^{n}-\left(\left(x+c^{x}\right)^{c r}\right)^{n} \in 2$. This implies that $\left(c^{2 n}\right)^{n-1} c^{r} x \in Z$. So $0=c^{(2 n-1) r} x c^{r}=c^{r} e^{(2 n-1) r} x$, and 
hence $c^{8} x=0$ for a positive integer $B$. Thus $x \in B$, and $A \subset B$. Similarly, $B \subset A$. So $A=B$ and hence $A$ is an ideal of $R$. Since $A$ is a nonzero ideal of $R, b^{t} \in A$ for some $t \geq 1$. Thus $b^{t} c^{r}=0$ for some $r \geq I$, and since $c$ is not nilpotent, then $b^{t}$ is a zero divisor. Now we can repeat the above argument to show that the set $c=\left\{x \in R:\left(b^{t}\right)^{u} x=0\right.$ for some $\left.u \geq 1\right\}$ is an ideal of $R$. Since $c^{r} \neq 0 \in C, C \neq 0$, and hence $b^{k} \in C$ for some $k \geq 1$. So $b^{t u} \cdot b^{k}=0$. This contradicts the fact that $b$ is nonnilpotent. This proves claim $l$.

ClAIM 2. $R$ has no nonzero nilpotent elements.

To prove Claim 2, suppose that $u^{2}=0$ with $y \neq 0$. Then every element of $y R$ is a zero divisor, and hence by Claim 1 every element of $y R$ is nilpotent. Thus $y R$ is a nil right ideal, and so

$$
y R \subset J
$$

If $z=0$, then by hypothesis, for every $c, d$ in $R$ there exists an integer $n=n(c, d) \geq 1$ such that $(c d)^{n}=(d c)^{n}$, which implies by [2] that $R$ is commutative. So we may assume that $Z \neq 0$, and let $0 \neq z \in Z$. Since $R$ is prime and $0 \neq z \in Z$, then

$$
z \text { is not a zero divisor, } 0 \neq z \in Z \text {. }
$$

Let $a \in J$. Using (1) with $(y+z)$ instead of $x$, there exists an integer $n \geq 1$ such that

$$
\left(a(y+z)^{2 n}-(y+z)^{2 n} a\right) a=a\left(a(y+z)^{2 n}-(y+z)^{2 n} a\right) .
$$

Since $y^{2}=0$ and $z \in Z$, (4) implies

$$
\left[a\left(2 n z^{2 n-1} y+z^{2 n}\right)-\left(2 n z^{2 n-1} y+z^{2 n}\right) a, a\right]=0 \text {, }
$$

and hence

$$
2 n z^{2 n-1}[[a, y], a]=0 \text {, }
$$

and since $R$ is torsion-free and $z^{2 n-1}$ is not a zero divisor

$$
[[a, y], a]=0 \text { for all } a \in J, y^{2}=0 \text {. }
$$


Using induction on the index of nilpotence of nilpotent elements $v$ and proceeding as above yields that

$$
\begin{aligned}
& \qquad[a, v], a]=0 \text { for all } a \in J \text {, and all nilpotents } v \text {. } \\
& \text { Since } y R \text { is nil, (2) and (6) imply that }
\end{aligned}
$$$$
[[a, v], a]=0 \text { for all } a, v \text { in } y R \text {. }
$$

Hence $y R$ is a nil right ideal satisfying a polynomial identity. So by Lemma 2.1.1 of [4], $R$ has a nonzero nilpotent ideal, a contradiction. Hence $y R=0$, and so $y x=0$ for all $x \in R$. Thus every element of $R$ is a zero divisor, and hence nilpotent by Claim 1 . This is a contradiction since $R$ has no nonzero nil ideals. Thus $y=0$ and Claim 2 is now proved.

Now we can complete the proof of Theorem 2. By Lemma 3, for each $a \in J, x \in R$, there exist integers $n=n(x, a) \geq 1$ and $m=m(x, a) \geq 1$ such that $(4 m) !\left[a, x^{2 n}\right]^{4 m}=0$. Using Claim 2, and that $R$ is torsion-free, we get $\left[a, x^{2 n}\right]=0$. Thus for every $x, a \in J$ there exists an integer $n=n(x, a) \geq 1$ such that $\left[a, x^{2 n}\right]=0$, and hence $J$ is commutative [3]. $R$ is prime, and has a commutative nonzero ideal $J$, hence $R$ is commutative [4]. This completes the proof of Theorem 2.

\section{References}

[1] Hazar Abu-Khuzam and Adil Yaqub, "A commutativity theorem for division rings", Bull. Austral. Math. Soc. 21 (1980), 43-46.

[2] L.P. Belluce, I.N. Herstein and S.K. Jain, "Generalized commutative rings", Nagoya Math. J. 27 (1966), 1-5.

[3] I.N. Herstein, "A comutativity theorem", J. Algebra 38 (1976), $112-118$.

[4] I.N. Herstein, Rings with involution (University of Chicago Press, Chicago, Illinois; Iondon; 1976).

Department of Mathematical Sciences, University of Petroleum and Minerals, Drahran, Saudi Arabia. 